\title{
Biologic significance of angiopoietin-2 expression in human hepatocellular carcinoma
}

\author{
Shinji Tanaka, ${ }^{1}$ Masaki Mori, ${ }^{1}$ Yoshihiro Sakamoto, ${ }^{2}$ Masatoshi Makuuchi, ${ }^{2}$ \\ Keizo Sugimachi, ${ }^{3}$ and Jack R. Wands ${ }^{4}$ \\ ${ }^{1}$ Department of Surgery, Medical Institute of Bioregulation, Kyushu University, Beppu 874-0838, Japan \\ ${ }^{2}$ Department of Surgery, Faculty of Medicine, University of Tokyo, Tokyo 113-0033, Japan \\ ${ }^{3}$ Department of Surgery II, Faculty of Medicine, Kyushu University, Fukuoka 814-8582, Japan \\ ${ }^{4}$ Molecular Hepatology Laboratory, Cancer Center, Massachusetts General Hospital, Harvard Medical School, \\ Boston, Massachusetts 02114, USA
}

Address correspondence to: Shinji Tanaka, Department of Surgery, Medical Institute of Bioregulation, Kyushu University, 4546 Tsurumibaru, Beppu 874-0838, Japan. Phone: 81-977-27-1650; Fax: 81-977-27-1651; E-mail: shinji@tsurumi.beppu.kyushu-u.ac.jp

Received for publication August 12, 1998, and accepted in revised form December 7, 1998.

\begin{abstract}
Human hepatocellular carcinoma (HCC) is generally a highly vascular tumor, but the mechanisms of neovascularization that permit rapid growth have not been defined. Angiopoietins (Ang) recently have been identified as ligands for vascular endothelial-specific Tie2 receptor tyrosine kinase and may be important growth factors in the generation of new blood vessels. We investigated Ang expression in 23 samples of HCC and paired adjacent uninvolved liver samples to determine if these genes have a potential role in the growth and spread of this disease. The full coding sequence of a variant angiopoietin-2 (Ang2) cDNA was obtained from HCC specimens, and the biologic consequences of overexpression on tumor formation and hemorrhage were determined in an animal model system. Angiopoietin-1 (Ang1) was equally expressed in HCC and adjacent noncarcinomatous liver tissue. Surprisingly, Ang2 was found to be highly expressed only in tumor tissue. In addition, Ang2 was expressed in 10 of 12 hypervascular HCC, but only in 2 of 11 hypovascular HCC. Ectopic expression of Ang2 in nonexpressing HCC cells promotes the rapid development of human hepatomas and produces hemorrhage within tumors in nude mice. These results suggest a role for Ang2 in the neovascularization of HCC. This enhanced gene expression may contribute to the clinical hypervascular phenotype, as well as tumor formation and progression.
\end{abstract}

J. Clin. Invest. 103:341-345 (1999).

\section{Introduction}

The natural history of hepatocellular carcinoma (HCC) is generally one of rapid progression, with increasing pain, hepatomegaly, jaundice, weight loss, and ascites formation as the predominant clinical features. The cause of death may be catastrophic, with spontaneous rupture of the tumor and massive intraperitoneal bleeding due to the hypervascular characteristics of many such tumors. However, the factors responsible for the neovascularization process and subsequent growth and spread of this disease have not been identified.

Neovascularization involves the sprouting of new blood vessels from preexisting ones and is essential for tumor development and progression (1). In this regard, neovascularization will support tissue growth by providing oxygen and nutrients. Angiogenic factors are required not only for the development of tumors but also for expansion and metastatic spread of malignant cells. Such events represent a complex process and involve (a) degradation of the periendothelial matrix and support cells around preexisting vessels, $(b)$ migration of endothelial cells toward the angiogenic stimuli, and $(c)$ proliferation and formation of new microvessels. Previous studies suggest that several growth factors and cytokines specific for vascular endothelial cells are required to induce migration and proliferation of these cells both in vitro and in vivo $(1,2)$.
Recently, a novel family of angiogenic factors, designated as angiopoietins (Ang), has been identified by Yancopoulous and colleagues $(3,4)$. Ang family proteins have been shown to function as ligands for the Tie $2 /$ Tek vascular endothelial-specific receptor $(5,6)$. Because the Tie2 receptor regulates endothelial interactions with periendothelial support cells and is required for vascular maturation to occur (7-9), Ang may play an important role in vascular morphogenesis and maintenance between the endothelium and supporting tissues. HCC is a tumor frequently associated with increased vascularity (10). Indeed, spontaneous rupture of the tumor may be observed during the clinical course of disease. In this study, we identified expression of a specific Ang family member that is closely associated with hypervascular tumors. Furthermore, stable transfection of this gene into nonexpressing HCC cells resulted in striking tumor development and extensive hemorrhage when grown as solid tumors in nude mice, leading to a high mortality rate in these animals. These studies suggest a novel role for this angiogenic factor in the development of hypervascular HCC.

\section{Methods}

Patients. We studied 23 patients with resectable HCC. Tumor size varied from $1.8 \mathrm{~cm}$ to $14.5 \mathrm{~cm}$, and all individuals had a single tumor. At the time of surgery, wide resection margins allowed us to obtain enough tissues to compare gene expres- 
sion in tumor tissue versus the uninvolved normal hepatic counterpart. There were 14 males and 9 females with HCC tumors. Age varied from 47 to 77 years, with a mean age of 60 years. Of these 23 patients, 12 individuals had liver cirrhosis and 9 others had chronic hepatitis without cirrhosis. The hepatitis $\mathrm{B}(\mathrm{HBV})$ - and hepatitis $\mathrm{C}(\mathrm{HCV})$-associated viral etiology of the $\mathrm{HCC}$ were as follows: $\mathrm{HCV}^{+} \mathrm{HBV}^{-}: n=15 ; \mathrm{HCV}^{+} \mathrm{HBV}^{+}: n$ $=2 ; \mathrm{HCV}^{-} \mathrm{HBV}^{+}: n=4 ; \mathrm{HCV}^{-} \mathrm{HBV}^{-}: n=2$. Thus, $73.9 \%$ (17 cases) were $\mathrm{HCV}$-related (including both $\mathrm{HBV}^{+}$and $\mathrm{HCV}^{+}$) and $21.6 \%$ (6 cases) were HBV-related (including both $\mathrm{HBV}^{+}$and $\mathrm{HCV}^{+}$). The stated HCC vascularity was assessed by the intensity and extent of vessel staining as determined by preoperative angiography. Based on these criteria, tumors were subsequently broadly divided into hypovascular and hypervascular groups.

Analysis of Ang genes in HCC tumors. Total RNA was extracted using the acid guanidine phenol chloroform method followed by DNase treatment and reverse transcriptase (RT) reaction as described previously (11). To analyze expression of Ang family genes, the consensus sequences (YTL/ILPE and QQNAVQN) were used as reported by Maisonpierre et al. (4). In brief, RTPCR was performed for 25 cycles using the degenerated primers 5'-TAYACNHTNYTNCCNGA-3' and 5'-TTYTGNACNGCRTTYTGYTG- $3^{\prime}$ under the following conditions: denaturing at $94^{\circ} \mathrm{C}$ for $1 \mathrm{~min}$, annealing at $55^{\circ} \mathrm{C}$ for $1 \mathrm{~min}$, and polymerization at $72^{\circ} \mathrm{C}$ for $1 \mathrm{~min}$. The PCR products were extracted and directly sequenced or subsequently cloned for sequence analysis using a Cycle Sequencing kit (Applied Biochemical Inc, Foster City, California, USA). The RNA expression was analyzed by RT-PCR using the following primers as described previously (12): Ang 1: 5'-AAATGGAAGGAAAACACAAGGAA-3' and 5'ATCTGCACAGTCTCTAAATGGT-3' (annealing at $51^{\circ} \mathrm{C}$,

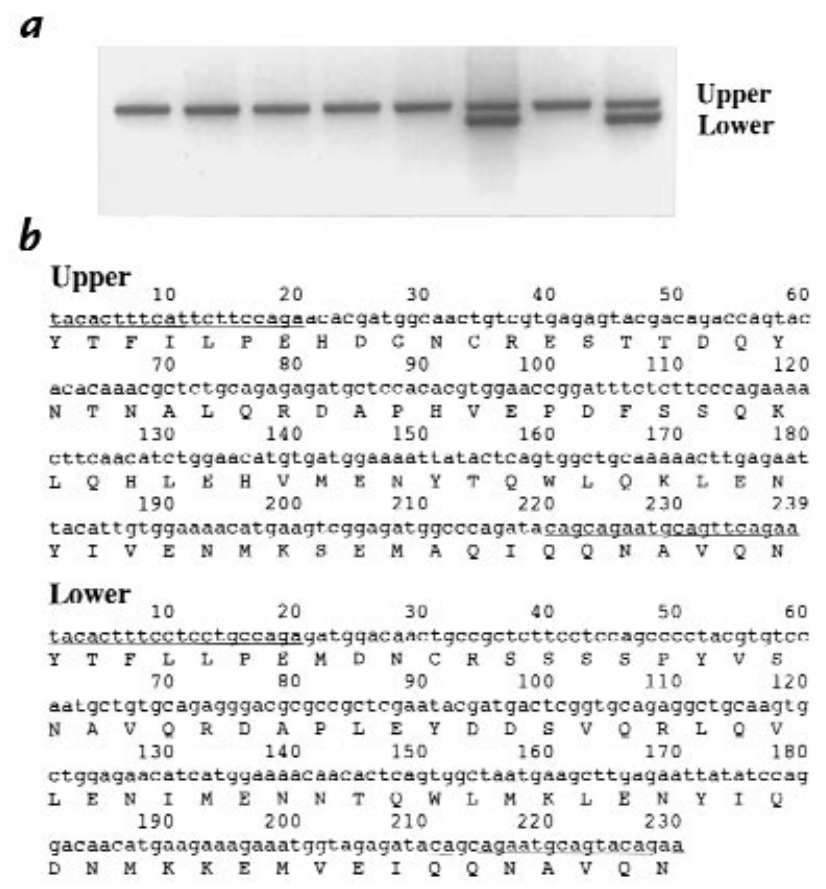

\section{Figure 1}

Expression of Ang family genes in hypovascular and hypervascular HCC. (a) Degenerate PCR analysis using the Ang consensus sequences (YTL/ILPE and QQNAVQN) revealed two distinct fragments in hypervascular HCC ( $3 T$ and $4 T)$. The lower band was not present in hypovascular $\mathrm{HCC}(1 T$ and $2 T)$ and adjacent noncarcinomatous liver tissue $(1 \mathrm{~N}, 2 \mathrm{~N}$, $3 \mathrm{~N}$, and $4 \mathrm{~N}$ ). (b) Sequence analysis of the 239-bp and 230-bp fragments. The upper and lower bands were found to be identical to Ang1 and Ang2, respectively. Ang, angiopoietin; HCC, hepatocellular carcinoma. product size $=266 \mathrm{bp}$ ); Ang2: 5'-GACGGCTGTGATGATAGAAATAGG-3' and 5'-GACTGTAGTTGGATGATGTGCTTG$3^{\prime}$ (annealing at $53^{\circ} \mathrm{C}$, product size $=264 \mathrm{bp}$ ). As a control, we performed RT-PCR of glyceraldehyde-3-phosphatase dehydrogenase (GAPDH) as reported previously (11): 5'-GTCAACGGATTTGGT-CTGTATT-3', 5'-AGTCTTCTGGGTGGCAGTGAT-3' (product size $=560$ bp). The primers 5'-AAGCTGCTGGTTTATTACTGAAGA-3' and $5^{\prime}$-GGATGTTTAGAAATCTGCTGGTC-3' (annealing at $54^{\circ} \mathrm{C}$ ) were used for isolation of the full coding sequence of Ang2. All PCR primers were selected to span the introns to detect specific mRNA sequences.

Biologic effects of ectopic expression of Ang 2 cDNA in HCC cells. The HCC-derived Ang2 cDNA was subcloned into the pcDNA3 vector and subsequently transfected into a human HuH7 HCC cell line as described (12). HuH7 cells express Ang1 mRNA but have no detectable endogenous Ang2 mRNA expression (see Fig. $3 a$ ). The expression of Ang mRNA was analyzed by RNase protection assays performed using the RPA II kit (Ambion Inc., Austin, Texas, USA) according to the manufacturer's protocol. In brief, a RT-PCR was performed to amplify Ang1 cDNA using the specific primers (5'-CAGCGCCGAAGTCCAGAAAAC-3', 5'-CTTCAACATCTGGAACATGTG-3') or for Ang2 using primers (5'-TAATAACTTACTGACTATGATG-3', 5'-CGTTAACATTCCCTAATTCTA-3'). This fragment was subcloned into the pcR2 vector to construct ${ }^{32} \mathrm{P}$-labeled antisense cRNA probe. This reagent was used to protect a $204 \mathrm{bp}$ for Ang1 or a 152 bp for Ang2, respectively. Approximately $40 \mu \mathrm{g}$ of total RNA was used for an overnight hybridization with the labeled cRNA probes at $42^{\circ} \mathrm{C}$ and then subsequently treated with RNase A and T1. The protected double-stranded RNA fragments were then analyzed by electrophoresis. After selection of clones in the presence of $800 \mu \mathrm{g} / \mathrm{ml}$ of G418, RNA was extracted and treated with DNase, and the expression levels of Ang 2 mRNA in each clone were determined by the RNase protection assays.

To investigate the tumorigenicity and biologic effects of Ang2 expression in HuH-7 cells, we injected nude mice (4-week-old females) with $10^{4}$ cells in $50 \mu$ l of PBS intraperitoneally near the liver bed as described previously (13). Nude mice ( $\mathrm{N}-20)$ were observed every day and analyzed for histological examination of tumor growth at 3 weeks after inoculation of cells or immediately after death of the animals. Tumor specimens were analyzed for RNase protection assays to detect Ang mRNA or fixed with $10 \%$ neutral buffered formalin and embedded in paraffin. The tissue blocks were cut to $5 \mu \mathrm{m}$, removed from the paraffin, then stained with hematoxylin and eosin for histological analysis. Four HuH7 clones stable-expressing Ang2 were evaluated for intraperitoneal tumor growth and hemorrhage using this biologic assay system.

\section{Results}

Expression of Ang family genes was analyzed by RT-PCR in HCC tumor-normal, liver-paired specimens with different vascularization patterns. Figure $1 a$ shows that the degenerate PCR amplified two cDNA fragments $239 \mathrm{bp}$ and $230 \mathrm{bp}$ in length from human liver and tumor tissue. The upper band ( $239 \mathrm{bp}$ ) was equally detected in all HCC tumors and adjacent normal liver, whereas the lower band (230 bp) represented a specific transcript found only in HCC tumors. Sequence analysis identified the upper DNA fragment as Ang1 and the lower as Ang2 (Fig. 1b). Ang1 and Ang2 expression was then analyzed in 23 HCC tumors by the specific RT-PCR. Preoperative angiography had classified 12 tumors as hypervascular and the remaining 11 tumors as hypovascular, as revealed by the vascular 
staining patterns. We found Ang1 expression in all HCC tumors and adjacent uninvolved normal liver tissues; there was no difference in the level of expression between the tumor and normal liver in these paired samples by semiquantitative PCR. In contrast, Ang2 expression was identified in 10 hypervascular HCCs (83.3\%), but in only 2 hypovascular HCCs $(18.2 \%)$ ( $P<0.05$, Fisher's exact test). More important, we found no detectable Ang2 mRNA by RT-PCR analysis in the adjacent noncarcinomatous liver tissue. The results suggest a close association between Ang2 expression and a hypervascular pattern in HCC.

The full coding sequence of Ang2 was obtained from four HCC tumors. Subsequent sequence analysis revealed that the Ang2 cDNAs derived from these tumors were identical to the sequence determined by Maisonpierre et al. (4), except that the HCC cDNA carries a short three-base deletion at nt position 802-804 in the coding sequence. This mutation leads to the deletion of a single alanine residue at position 268 of the amino acid sequence. The full cDNA sequence has been deposited with GenBank (accession No. AB008789).

In this regard, the biologic effect of ectopic Ang2 expression in a human HuH7 HCC cell line that expresses Ang1, but no detectable Ang2 transcripts, was assessed (Fig. 2a). Four-HuH7 independent clones (Ang2-V1, Ang2-V2, Ang2-W1, and Ang2-W2) were isolated and analyzed for gene expression (Fig. 2a). Analysis of growth curves of transfected cells demonstrated that cell proliferation was not affected by Ang2 expression (Fig. $2 b$ ). Next, to investigate the phenotype of Ang 2 transfectants in vivo, $10^{4}$ cells were injected intraperitoneally near the liver bed of each animal as described (13). Surprisingly, all 20 nude mice inoculated with HuH7-Ang2 clones died from extensive intraperitoneal bleeding within three weeks, as demonstrated in Fig. $3 a$. At the time of death, all 20 mice had developed large tumors (Fig. 3b), and significant hemorrhage within the tumors and into the peritoneum was observed (Fig. 3c). There was no hemorrhage or tumor development in the adjacent murine liver (Fig. $3 d$ ). The expression of the Ang2 gene was apparent in all tumors derived from HuH7-Ang2 clones as determined by RNase protection assays (Fig. $3 e$ ). In contrast to animals that received $\mathrm{HuH} 7$-Ang2 clones, all 20 nude mice inoculated with parental or mock DNA-transfected HuH7 cells survived, and no intraperitoneal tumor growth was observed at three weeks (Fig. 4). These results suggested a pathogenic role of Ang2 expression in the establishment and growth of hypervascular HCC tumors.

\section{Discussion}

One of the most common and devastating malignant tumors in the world is HCC. The major risk factors for the development of HCC are now well recognized, and some of the steps involved in the molecular pathogenesis of HCC have been elucidated in recent years. However, while important advances in understanding the sequence of events involved in the pathogenesis of other human cancers have been made, our understanding of how the multiple factors involved in hepatic oncogenesis operate and interact at the molecular level is far from complete (14). There is no information on the factors that allow for rapid HCC growth, partic-

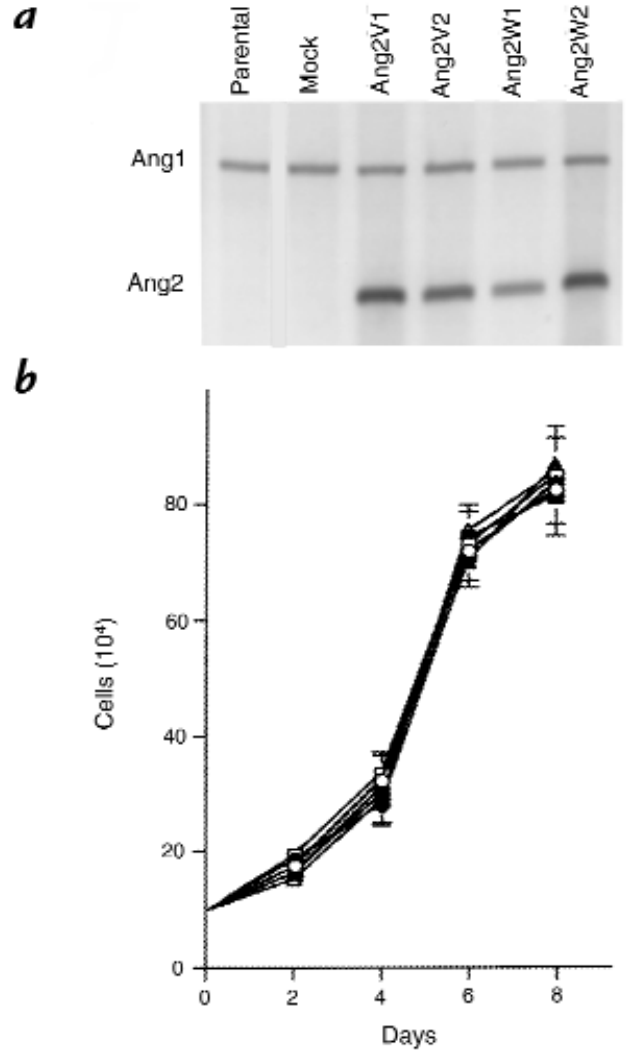

Figure 2

Growth of HuH7 HCC cells transfected with an Ang2 cDNA. (a) Expression of Ang1 and Ang2 in Ang2-transfected HuH7 clones (V1, V2, W1, and W2) compared with parental and mock-transfected HuH7 cells, as demonstrated by the RNase protection assays. (b) Curves illustrating the growth of $\mathrm{HuH} 7$ clones. Open circles: parental HuH7 cells; closed circles: mock-transfected HuH7 cells; open squares: HuH7-Ang2-V1 cells; closed squares: HuH7-Ang2-V2 cells; open triangles: HuH7-Ang2-W1 cells; closed triangles: HuH7-Ang2-W2 cells.

ularly with respect to the neovascularization process. Thus, we explored the potential role of the Ang family of genes in the pathogenesis of these tumors.

Transcription of the Ang2 gene has been shown to be quite restricted in adult tissues $(3,4)$. In contrast, Ang1 and Ang2 are widely expressed in many embryonic tissues. It interesting that Ang1 expression persists widely in adult tissues, whereas Ang2 appears to be selectively expressed only in the ovaries, uterus, and placenta. These organs are known to have extensive vascularization patterns under physiologic conditions, and this finding suggests a possible association of Ang2 expression with angiogenesis. Another clue for the role of Ang in angiogenesis is provided by levels of expression observed during ovulation (4). For example, in early follicle development, when the vasculature is quiescent, Ang1, but not Ang2, has been found to be expressed. In the late preovulatory follicles and postovulatory corpus luteum, where angiogenesis is quite active, the Ang2 gene is upregulated in the context of continuous expression of Ang1. These findings strongly suggest that expression of Ang2 may be required for vascular remodeling during angiogenesis. 

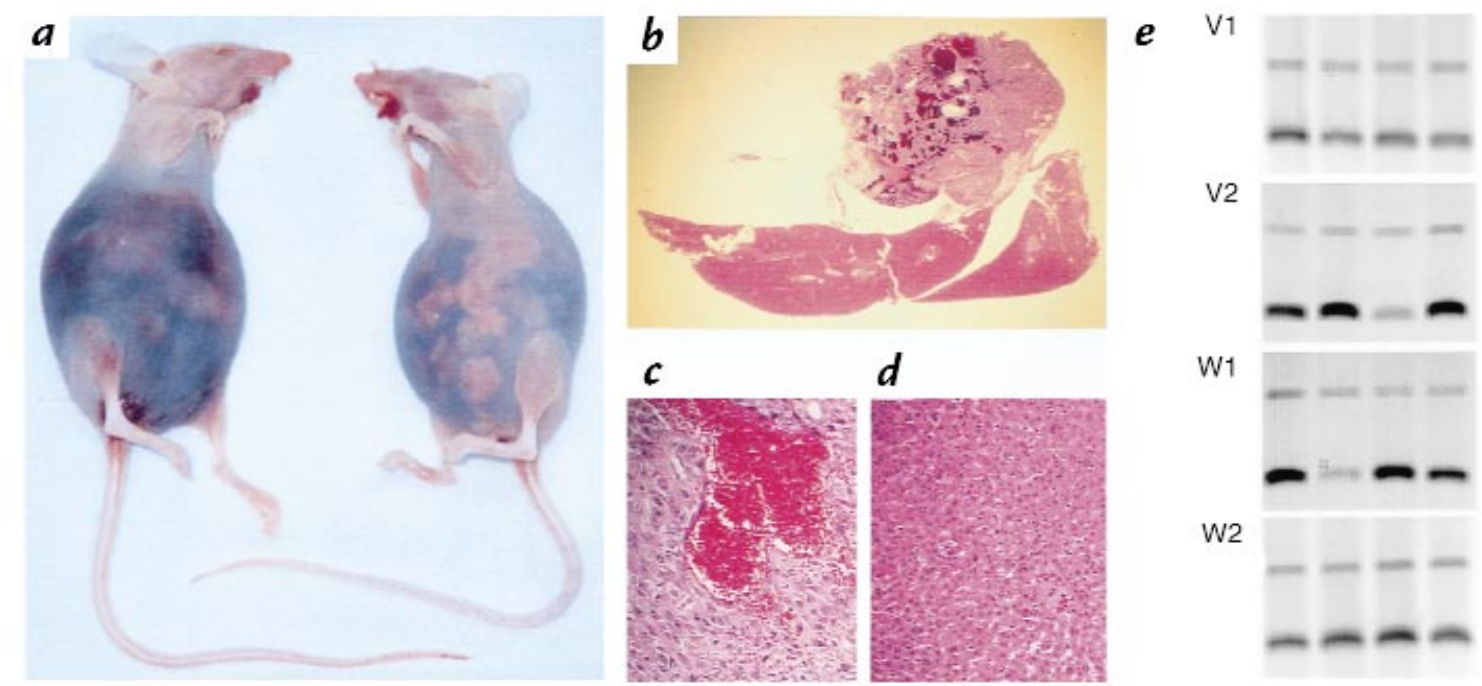

\section{Figure 3}

Characteristic of tumor growth in nude mice after intraperitoneal inoculation with $10^{4}$ Ang2-transfected HuH7 HCC cells. (a) Demonstration of the intraperitoneal bleeding phenomenon observed in nude mice at 15 days after inoculation with HuH7-Ang2-V1 (left) or HuH7-Ang2-W1 (right) cells. (b) Representative example of HCC tumor development in a nude mouse inoculated with HuH7-Ang2-V1 cells (hematoxylin and eosin staining). $\times 40$. (c) Hemorrhage was observed in these HCC tumors. $\times 100$. (d) Adjacent murine liver surrounding the HCC was free of hemorrhage and tumor and is presented for comparison. (e) Expression of Ang1 (upper bands) and Ang2 (lower bands) in four tumors derived from the HuH7-Ang2 clones (V1, V2, W1, and W2), as measured by the RNase protection assays.

We found that the variant Ang2 cDNA cloned from human HCC tumors has a coiled- coil (59-254 aa) and fibrinogen-like (280-466 aa) domains, and these regions are highly conserved among the Ang family of proteins (data not shown). It is noteworthy that Davis et al. (3) reported a similar variant of Ang1, where a three-base deletion resulted in the elimination of glycine at position 269. Sequence alignments among Ang family proteins reveal two nonconserved regions: an $\mathrm{NH}_{2}$-terminal hydrophobic region showing typical secretory signal sequence and a region spanning the coiled-coil and fibrinogen-like domains. Cysteine residues were also found to be highly conserved between Ang1 and Ang2 proteins, except for one cysteine residue in the spacer region between the conserved domains of Ang1 that was absent in the Ang2 protein $(3,4)$. Both the variable glycine 269 of Ang1 and Ala 268 of Ang2 were located in the variable spanning region between the two conserved domains. Therefore, there is little doubt that Ang2 is highly expressed in hypervascular HCC, and further studies are needed to determine if other Ang2 gene variants are present in such tumors.

The in vivo biologic effects of enhanced Ang2 expression in HCC tumors with respect to promoting angiogenesis and hemorrhage has been demonstrated in this study using an animal model system. Ang1 has been isolated and shown to be a ligand for endothelial-specific Tie2 receptors and will induce autophosphorylation of this receptor on the endothelial cells present in blood vessels. However, Ang1-induced phosphorylation of Tie2 does not affect endothelial growth - at least in cell culture. The possible function of Tie 2 receptors after activation has been recently elucidated by gene knockout experiments in mice. Tie2 (9) or Ang1 (15) knockout mice demonstrate similar immature vascularization patterns as well as abatement of matrix contracts and support cells with the vascular endothelium. It is important to note that Ang2 was initially isolated as a homologue of Ang1 and will bind to the Tie 2 receptor with similar affinity (4). However, Ang2 does not induce receptor phosphorylation. Rather, it competitively inhibits Ang1 function as an activator of Tie2 kinase, indicating that Ang2 behaves as an antagonist of Ang1 with respect to biologic effects on the endothelial cell. Indeed, overexpression of Ang2 in a transgenic mouse model revealed a loss of maturation of the vascular system, which is the identical phenotype observed with Tie2 or Ang1 knockout mice (4).

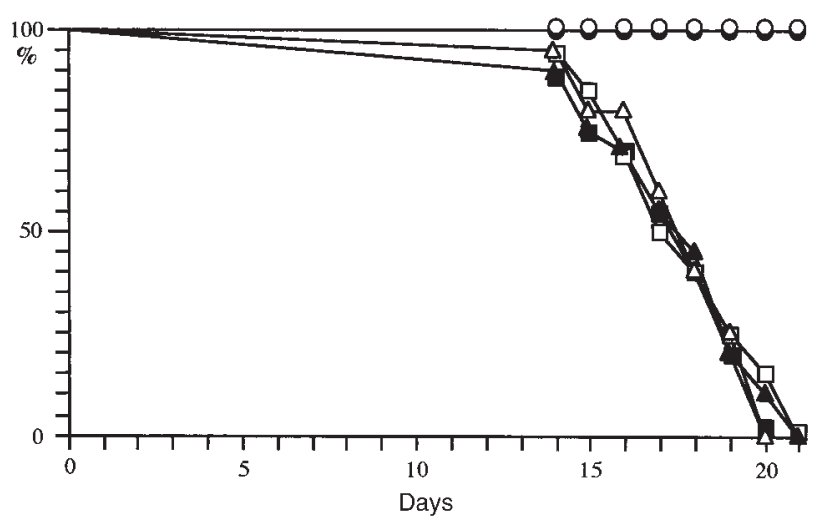

Figure 4

Survival rates of nude mice after intraperitoneal inoculation with $\mathrm{HuH} 7 \mathrm{HCC}$ clones. Open circles: parental HuH7 cells; closed circles: mock-transfected HuH7 cells; open squares: HuH7-Ang2-V1 cells; closed squares: HuH7-Ang2-V2 cells; open triangles: HuH7-Ang2-W1 cells; closed triangles: HuH7-Ang2-W2 cells. 
Therefore, based on these observations, it has been suggested that Ang1 activates Tie2 receptors on endothelial cells to promote recruitment and interaction with support cells such as pericytes, smooth muscle cells, and myocardiocytes. In contrast, Ang2 expression reduces matrix contacts and interactions of support cells with endothelial cells, which are necessary for the neovascularization process to occur (16). Our findings suggest that Ang2 expression may be essential for vascular remodeling in HCC tumors to allow access and stimulation of vascular responsiveness by additional angiogenic inducers as hypothesized by Hanahan (17). Further study of signaltransduction pathways in vascular endothelial cells induced by Ang1 activation of the Tie 2 receptor in the context of Ang2 expression in HCC will be interesting (18-20). Analysis of such angiogenic signals may provide insights on how to prevent HCC neovascularization, as well as how to inhibit tumor development and progression (21).

\section{Acknowledgments}

We thank Yasuji Yoshikawa for pathological analysis, and Kazue Ogata and Toshiko Shimooka for technical assistance. This study was supported by the Research Fund for Japan Medical Association, the Japan Society for the Promotion of Science (Fujita Memorial), the Inamori Foundation, a Grant-in-Aid from the Ministry of Education, Science, Sports and Culture of Japan, and grant CA-35711 from the National Institutes of Health.

1. Hanahan, D., and Folkman, J. 1996. Patterns and emerging mechanisms of the angiogenic switch during tumorigenesis. Cell. 86:353-364.

2. Beck, L., Jr., and D'Amore, P.A. 1997. Vascular development: cellular and molecular regulation. FASEB J. 11:365-373.

3. Davis, S., et al. 1996. Isolation of angiopoietin-1, a ligand for the TIE2 receptor, by secretion-trap expression cloning. Cell. 87:1161-1169.

4. Maisonpierre, P.C, et al. 1997. Angiopoietin-2, a natural antagonist for Tie2 that disrupts in vivo angiogenesis. Science. 277:55-60.
5. Runting, A.S., Stacker, S.A., and Wilks, A.F. 1993. Tie2, a putative protein tyrosine kinase from a new class of cell surface receptor. Growth Factors. 9:99-105.

6. Dumont, D.J., Gradwohl, G.J., Fong, G.H., Auerbach, R., and Breitman, M.L. 1993. The endothelial-specific receptor tyrosine kinase, tek, is a member of a new subfamily of receptors. Oncogene. 8:1293-1301.

7. Vikkula, M., et al. 1996. Vascular dysmorphogenesis caused by an activating mutation in the receptor tyrosine kinase TIE2. Cell. 87:1181-1190.

8. Dumont, D.J., et al. 1994. Dominant-negative and targeted null mutations in the endothelial receptor tyrosine kinase, tek, reveal a critical role in vasculogenesis of the embryo. Genes Dev. 8:1897-1909.

9. Sato, T.N., et al. 1995. Distinct roles of the receptor tyrosine kinases Tie1 and Tie-2 in blood vessel formation. Nature. 376:70-74.

10. Liver Cancer Study Group of Japan. 1990. Primary liver cancer in Japan. Clinicopathologic features and results of surgical treatment. Ann. Surg. 211:277-287.

11. Tanaka, S., et al. 1998. A novel frizzled gene identified in human esophageal carcinoma mediates APC/ $\beta$-catenin signals. Proc. Natl. Acad. Sci. USA. 95:10164-10169.

12. Tanaka, S., and Wands, J.R. 1996. A carboxy-terminal truncated IRS-1 dominant negative protein reverses the human hepatocellular carcinoma malignant phenotype. J. Clin. Invest. 98:2100-2108.

13. Artega, C.L., et al. 1993. Anti-transforming growth factor (TGF)- $\beta$ antibodies inhibit breast cancer cell tumorigenicity and increase mouse spleen natural killer cell activity. J. Clin. Invest. 92:2569-2576.

14. Moradpour, D., and Wands, J.R. 1996. Hepatic incogenesis, IVD. Tumors of the liver. In Hepatology: a textbook of liver disease. Volume 2. 3rd ed. W.B. Saunders. Philadelphia, PA. 1490-1512.

15. Suri, C., et al. 1996. Requisite role of angiopoietin-1, a ligand for the TIE2 receptor, during embryonic angiogenesis. Cell. 87:1171-1180.

16. Folkman, J., and D'Amore, P.A. 1996. Blood vessel formation: what is its molecular basis? Cell. 87:1153-1155.

17. Hanahan, D. 1997. Signaling vascular morphogenesis and maintenance. Science. 277:48-50.

18. Huang, L., Turck, C.W., Rao, P., and Peters, K.G. 1995. GRB2 and SHPTP2: potentially important endothelial signaling molecules downstream of the TEK/TIE2 receptor tyrosine kinase. Oncogene. 11:2097-2103.

19. Jones, N., and Dumont, D.J. 1998. The Tek/Tie2 receptor signals through a novel Dok-related docking protein, Dok-R. Oncogene 17:1097-1108.

20. Kontos, C.D., et al. 1998. Tyrosine 1101 of Tie2 is the major site of association of p85 and is required for activation of phosphatidylinositol 3kinase and Akt. Mol. Cell. Biol. 18:4131-4140.

21. Fidler, I.J., and Ellis, L.M. 1994. The implications of angiogenesis for the biology and therapy of cancer. Cell. 79:185-188. 